\title{
1. LIC for Surface Flow Feature Detection
}

\author{
David L. Kao
}

\begin{abstract}
The Line Integral Convol ition (LIC) algorithm has received a lot of attention and interest. Yet, on $y$ a few of the current LIC related algorithms deal specifically with color textures for automatic detection of flow features. In this paper, I give a brief $r$ view of current work in this area.
\end{abstract}

\subsection{Problem}

In windtunnel experiments, s ientists are interested in studying the flow behavior during actual flow con fitions. A common technique is to inject smoke during the experiment and to analyze the flow behavior by observing the flow pattern formed by the smoke. Some scientists have analyzed the flow pattern near the surface of the test model by coating the body with a mixture of paint and oil and then examining the flow pattern generated by the paint/oil. The resulting flow pattern is commonly referred to as surface oil flou.

In numerical fow visualization, vector plots and streamlines have been used to simulate surface oil fiows. These techniques have provided useful insights about the surface flow. Lnfortunately, these techniques require the users to know a priori locations to compute the particles and vectors. Another disadvantage is that the resulting fow patterns are discrete, and the output image quality may be cluttered with dense vectors/streamlines. The LIC algorithm generates surface flow patterns that are very intuitive to understand and it does not have the disadvantages of the conventional vector plots and streamlines.

Good progress has been made with the LIC algorithm introduced by Cahral and Leedom [1.1] which produces synthetic texture patterns based on an input vector field. An excellent tutorial on LIC can be found in the course notes by $\mathrm{Ma}$ et al. [1.2. Because the LIC technique generates texture patterns that reveal the flow direction in the given grid domain, it is ideal for simulating surface oil flow; in CFD applications. Although the algorithm has been shown to be very fffective in revealing global How features, the monochrome LIC images generatell by the algorithm sometimes do not reveal How features clearly because these features are displayerl on a gray-scale texture background. Figure 1.1 depicts an image of surface flow computed 
using the LIC algorithm on a cylindrical body. The monochrome texture depicts interesting flow separation and reattachment lines along the cylindrical body. There are also several critical points, where the velocity is zero, near the nose of the cylindrical body. However, some of these critical points are not easily seen because of the monochrome texture background. Scientists would like to see these interesting features automatically highlighted along with the flow texture patterns.

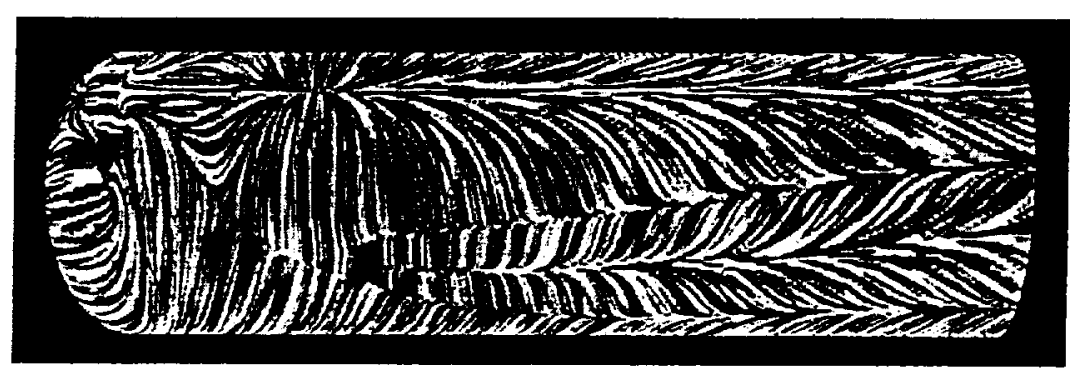

Fig. 1.1. Monochrome surface flow texture near the surface of a cylindrical body.

\subsection{Scalar Function}

A straightforward way to ennance flow textures is to color the texture by a selected scalar function. Srme common functions are pressure coefficient, velocity magnitude, and voricity magnitude. A particular scalar function may or may not highlight the How featurts. Furthermore. the color map uscd may not correlate well with the underlying flow texture patterns. For example, the color texture may not clearly delineate flow separations and reattachments. The top of Figure 1.2 depicts surface How colored by pressure coefficient. The input flow ficld is the same as the one used for Figure 1.1. Note that this scalar functio, highlights the critical point near the top of the cylindrical body but not those near the nose of the cylinderical body. Shown in the lower half of Fizure 1.2 is the flow texture colored by velocity magnitude. For this data set. the velocity matgnitude function gives a very good depiction of the separat on and reattachment lines and several critical points.

Figure 1.3 shows another comparison of a monochrome texture with scalar colored texture using a delta wing flow data set. The monochrome texture (upper left) does tot give a cle ar depiction of the interesting flow separations inti reattachments along the leading edge of the delta wing. The pressure coefficient texture (upper right) does not rival the flow fearures along the leatling edge of the wing. The velocity magnitude texture (lower left) gives 


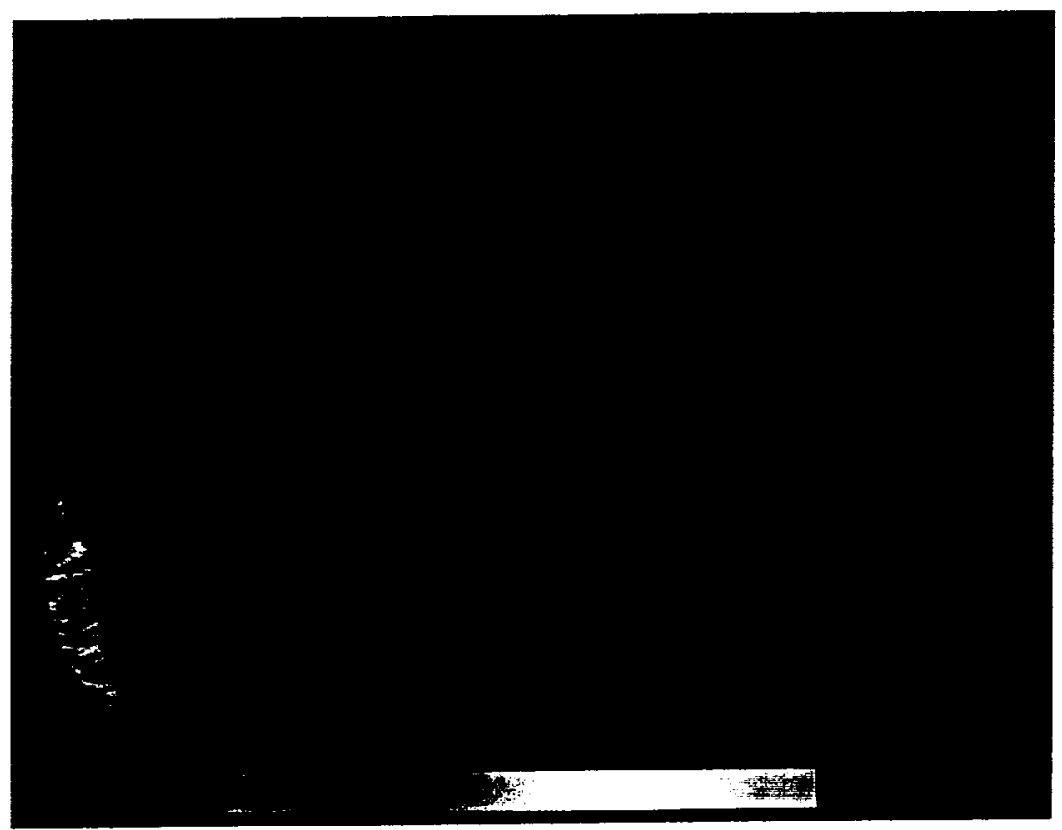

Fig. 1.2. Color surface flow texture based on pressure coefficient (top) and velocity magnitude (bottom). Lowest value is colored in blue and highest value is colored in red.

some correlation of the critical points (dark blue regions) and the flow separations and reattachments along the wing. However, due to the color map used, there are some misleading red streams near the nose tip of the wing even though the flow is quite steady in that region. Shown in the lower right of Figure 1.3 is the texture colored by vorticity magnitude. This scalar function seems to give the best correlation of flow features with color.

\subsection{Velocity Direction}

Another common method is to color the texture based on velocity direction. In a case study, Johannsen and Moorhead [1.3] used colors to represent flow directions in their study of ocean basin flow features. Using the HSV color model, they mapped the vector length to saturation and lightness. Boring and Pang [1.4] have also investigated mapping of velocity direction and magnitude to HSV value and hue. In their application, they used light sources to select and highlight flow regions with similar flow directions. We have found that the velocity direction is best represented using color when visualizing flow separation and reartachments [1.5]. Figure 1.4 shows surface flow colored by velocity direction. The lower half depicts a counterclock-wise circular flow colored by velocity direction. From the figure, it shows that horizontal velocity 


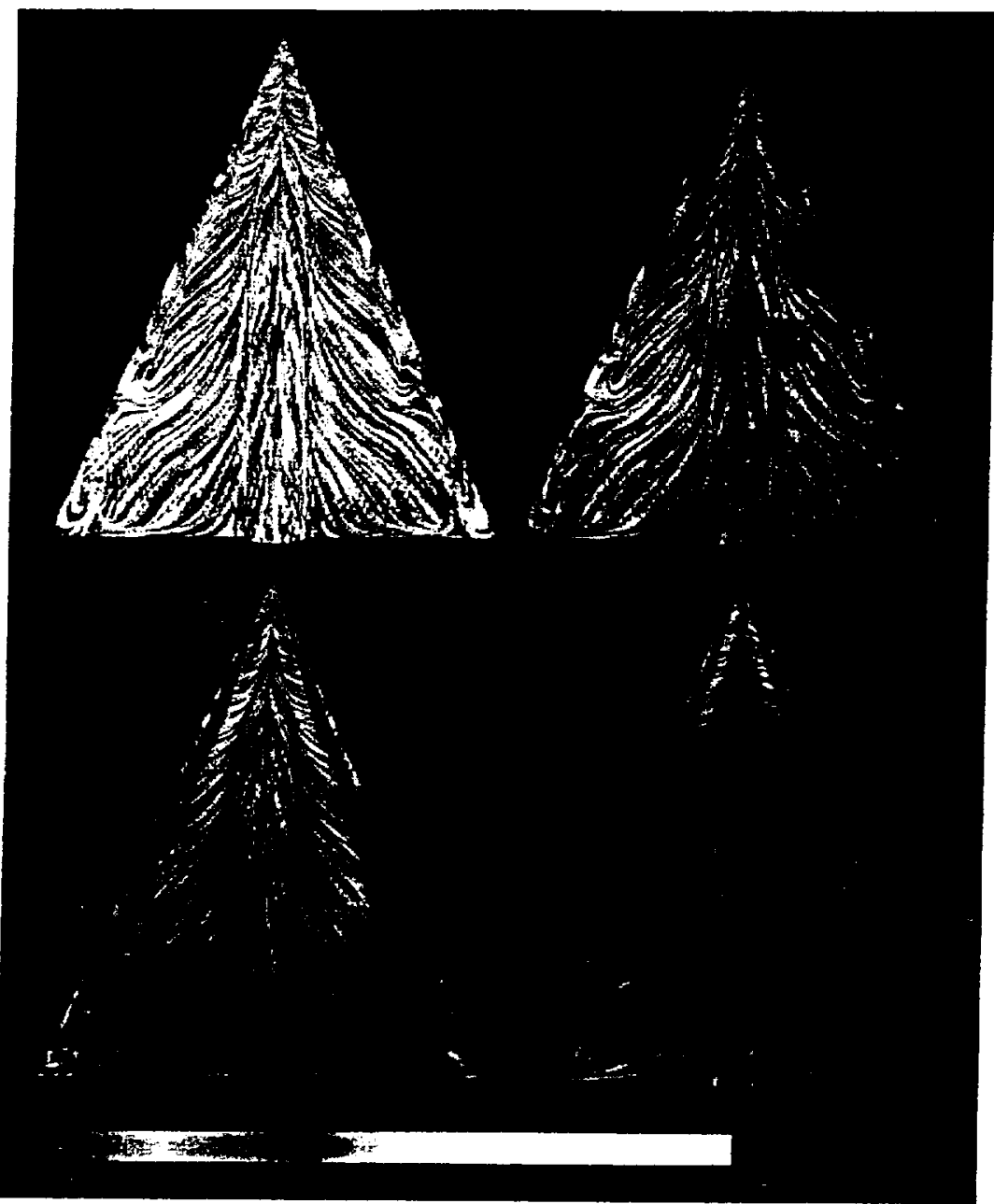

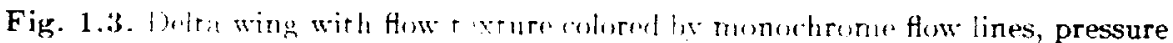

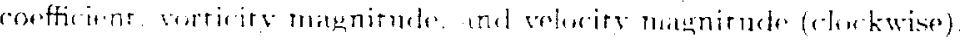

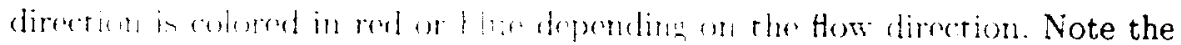

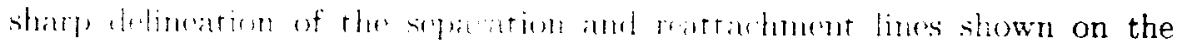

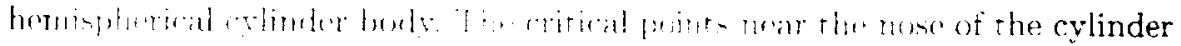

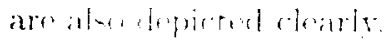

\subsection{Fin Separation ai d Pattachment}

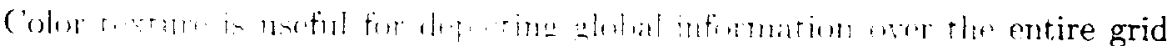

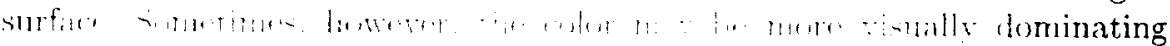




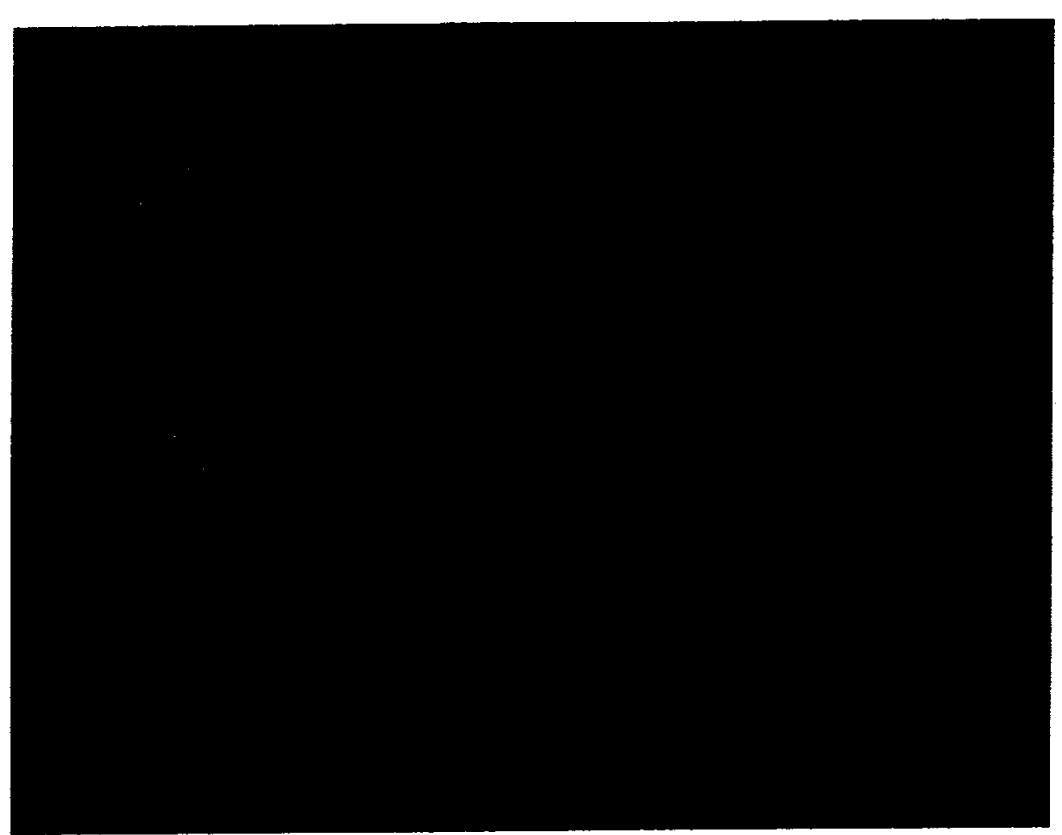

Fig. 1.4. LIC texture colored by velocity direction.

than the flow texture pattern. This is fine as long as the color change always highlights the flow features. But it is not guaranteed. One simple and fast solution is to color the texture only when the neighboring velocity changes significantly in direction and to keep the texture monochrome everywhere else. In flow separation, streamlines converge and then deflect from the surface of the body. This implies that the streamlines converge along the separation line from opposite directions. Analogously, when the flow is impinging on the surface, streamlines diverge along a reattachment line. By computing the angles formed by neighboring velocity vectors, we can check for the angles that are greater than some threshold value [1.5]. We then color the textures that meet this criterion. The algorithm proceeds as follows: At each texel $t(i, j)$, an average velocity vector $v_{a}(i, j)$ is computed based on the average of the velocity vector at $t(i, j)$ and its eight neighboring texels. Then, the velocity angle $v_{\text {ang }}(i, j)$ at $t(i, j)$ is set to the maximum of the dot products of $v_{a}(i, j)$ and the average velocity vectors from its neighboring texels. Let $v_{\text {ang }}(i, j)=\max \left(v_{a}(i, j) \bullet v_{a}(k, l)\right)$, where $k=i-1, i+1$ and $l=j-1, j+1$. The velocity angle $r_{a n g}(i, j)$ is then mapped to a color value. Hence, by selecting texels with velocity angles exceeding a threshold, one will only color textures where the flow direction changes rapidly. Shown in the upper half of Figure 1.5 is the LIC texture colored by velocity angle. Note that the color highlights the regions where flow separation and reattachment occur. 
Lsing the velority angle algorithm described above. flow separation and reattachment lines are both colored in the same color. It is possible to automatically distinguish between these two rases. Flow separations occur when the flow is learing the surfac: and when the fiow is impinging on the surface, it creates How attachment.s Hence by examining the velocity component normal to the grid surface, we can attempt to classify separation and reattachment [1.5]. In the lowe: half of 1.5. separation regions are colored in green, and the reattachment regions are colored in dark red.

Flow separation and reatiannum lines are even nore easily distinguished by using the two technicues in this section than when they are colored by a scalar function. Figure 1.6 c mpares the three techniques: colored by velocity direction. velocity angle, and separation and reattachment line classification using a delta wing data set. The separation and reatrachment line classification technique gives an immediate visual impression of the for features.

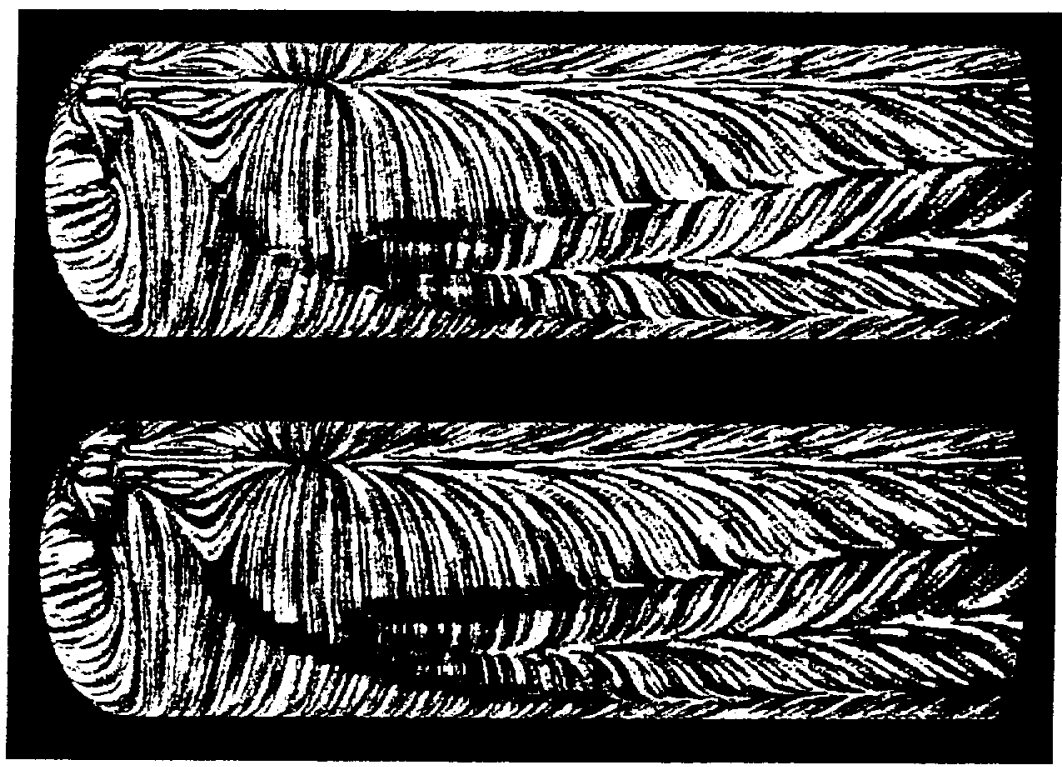

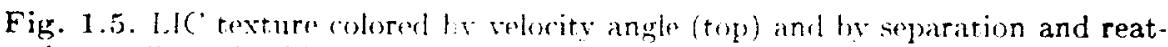
tachment lines alsisification (bortum).

\subsection{Validation}

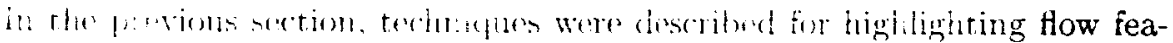
turtes antematically: There are serveral rexisting algorithms for computing crit-

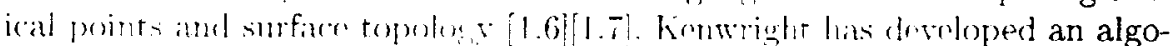

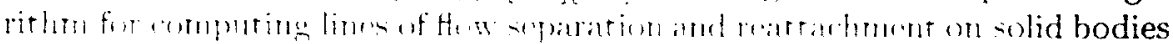


in a $3 D$ numerical flow fields. [1.8]. To validate the features that are highlighted by the separation and reattachment classification technique, we overlaid the results from these analytical algorithms on the flow texture. Figure 1.7 shows the surface flow topology, which is computed by the flow system developed by Globus et al. (top) [1.7], and the lines of separation and reattachment computed by Kenwright's algorithm (bottom) [1.8] overlaid on the texture computed by our algorithm [1.5].

Note that there is a very good match of the texture highlighted by our technique with the analytical separation and reattachment lines.

Figure 1.8 shows another validation using the delta wing data set.

\subsection{Future Direction}

Color LIC provides additional insight into the monochrome LIC texture patterns. The choice of scalar function used can change the global appearance of the flow texture. Sometimes, the change in color information is more dominant than the change in flow texture patterns. It is the user's judgment to choose the one that is most appropriate for their applications. Although this paper described some current techniques for automatic flow feature detection/highlighting, there is still a need for LIC algorithms that can provide diversified flow feature detection.

\subsection{Acknowledgments}

I would like to thank Randy Kaemmerer for proofreading this manuscript. I would atso like to thank Sam Uselton and Ravi Samtaney for their comments and suggestions. The delta wing dat set is courtesy of Neal Chaderjian, and the hemisphere cynlinder data set is courtesy of Susan Ying.

\section{References}

1.1 B. Cabral and C. Leedom (1993) Imaging vector fields using line integral convolution, Proceedings of ACM SIGGRAPH 93, August 1993. pp. 263-270.

12 K.-L. Ma, B. Cabral. H.-C. Hege, V. Interrante, and D. Stalling, (1997) Texture synthesis with Line Integral Convolution, Course Notes of ACM SIGGRAPH 97 , o. 8, August 1997 .

1.3 A. Johannsen and R. Moorhead (199-) Case study: visualization of mesoscale flow features in ocean basins, Proceedings of IEEE Visualization '94, October 1994, pp. 35j-358.

14 E. Boring and A. Pang (1996) Directional flow visualization of vector fields, Proceedings of IEEE Visualization '96, October 1996, pp. 389-392.

1.5 A. Okada and D.L. Kao (1997) Enhanced line integral convolution with flow feature detection, in Visual Data Exploration and Analysis IV, Proceedings of SPIE 3017. Feb. 1997, pp. 206-217. 


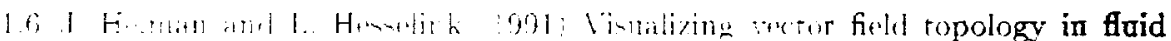

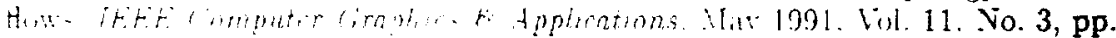

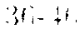

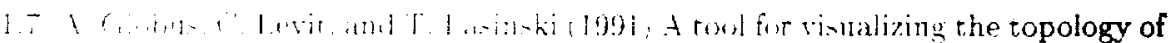

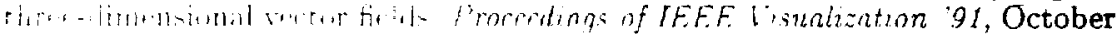
$159] \cdot 32.33-414$

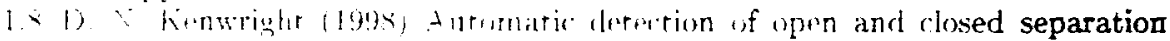
and atuhuntht limes. Pronerdings of IEEE Visualization 28. October 1998, [P] $1: 1-1, i x$

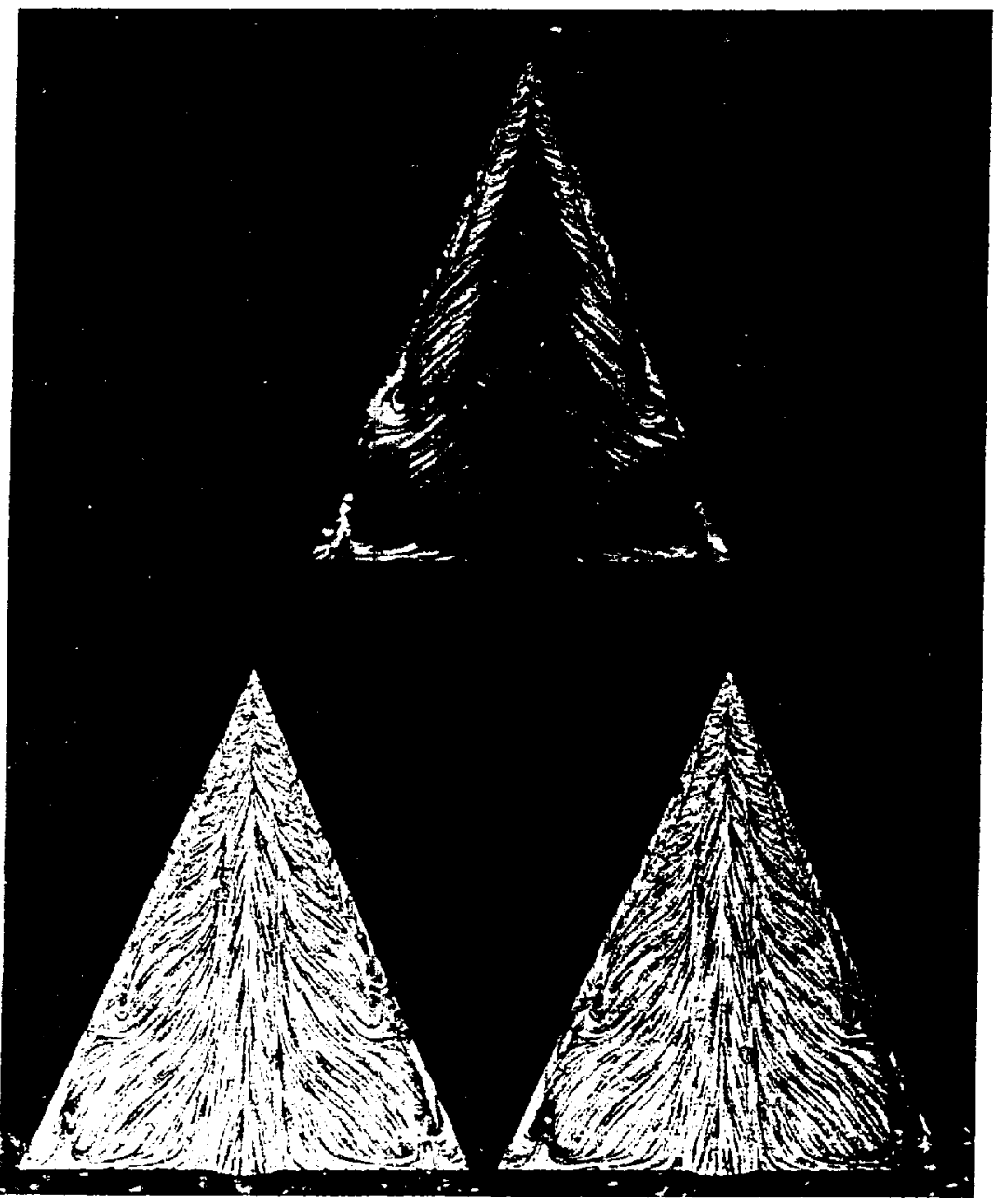

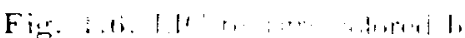

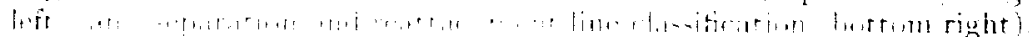




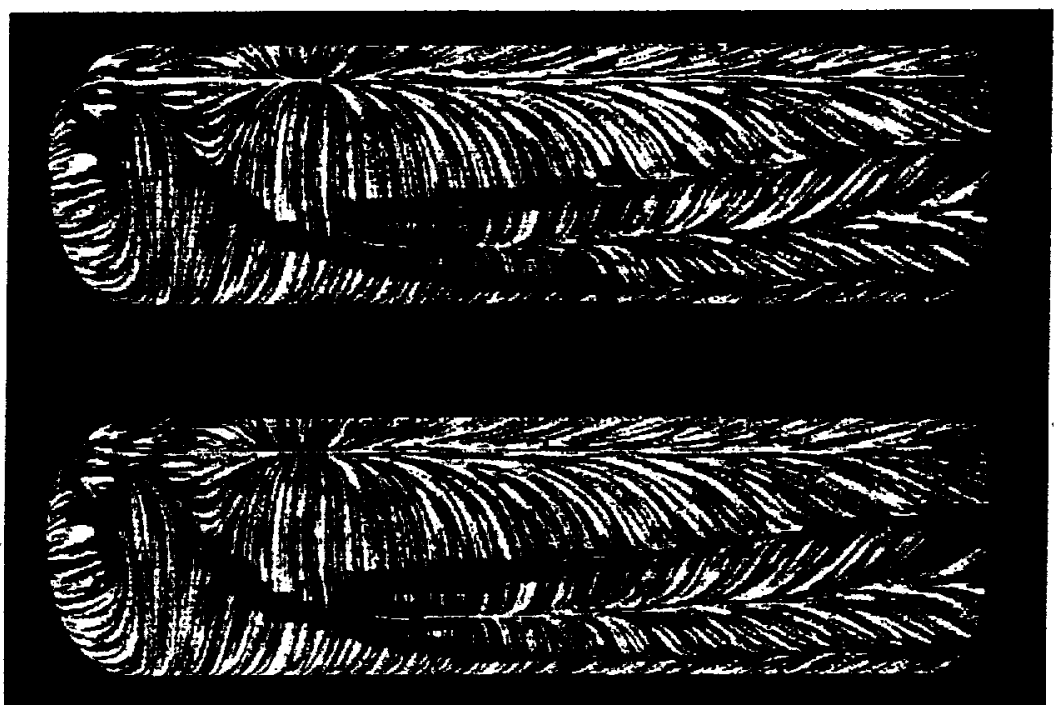

Fig. 1.7. Enhanced colsr LIC texture overlaid with surface topology (top) and analvtical lines of separation and reattachment (bottom).

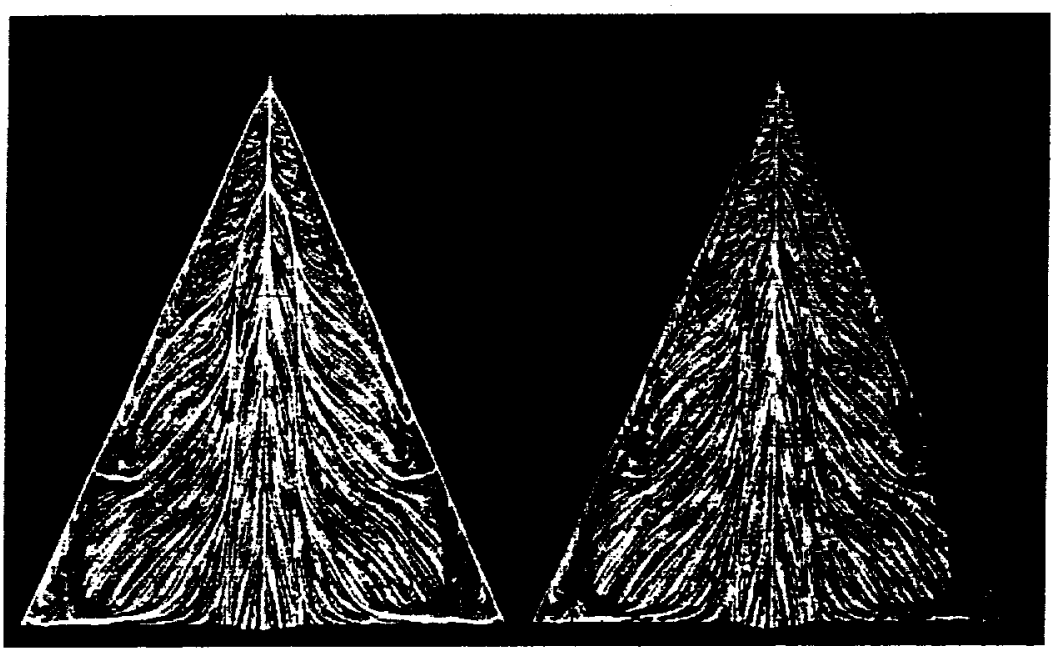

Fig. 1.8. Enhancel color IIC texture overlatd with surface topology (left) and analytical tines of separation and reattachment (righr). 